\title{
DE HEISENBERG Y GÖDEL A SAN AGUSTÍN, O DE LA INCERTIDUMBRE AL MISTERIO EDUCATIVO
}

Jorge Medina Delgadillo

Mens agitat molem. ("El espiritu mueve la materia").

Virgilio

\section{RESUMEN}

Los objetivos del presente escrito son, por una parte, manifestar la repercusión que han tenido las conclusiones de las ciencias físico-matemáticas del siglo XX en los terrenos educativos y, por otra, recuperar el pensamiento agustiniano como pista de solución a los problemas heredados en dichas conclusiones. Para delimitar el área de análisis del primer objetivo, recurriré a las conclusiones matemáticas de Kurt Gödel y a las de la física cuántica de Werner Heisenberg.

\section{INTRODUCCIÓN}

En los albores del vigésimo siglo, el mundo, y en especial, el mundo científico, se enriqueció por los avances que en las ciencias físico-matemáticas realizaron grandes científicos; entre otros, Born, Ackermann, Einstein, Minkowski, Rutherford, Heisenberg, Gauss, Hilbert, Planck, Gödel, propusieron una nueva forma de entender la realidad -forma no menos revolucionaria que la de Galileo y Copérnico en su tiempo-, cuyas consideraciones traspasaron el umbral de los números e incidieron en el pensamiento filosófico y, por ende, en el pensamiento educativo. 
Las teorías del caos y la complejidad, que actualmente se presentan como paradigma de comprensión de la realidad, nos obligan a revisar críticamente su origen y a considerar la validez o invalidez de sus conclusiones en el terreno humano. Ciertamente, los objetos de estudio de las ciencias físico-matemáticas se presentan al ser humano con cierta docilidad y pasividad manipulable, de ahí que los progresos en este ámbito del saber sean más vertiginosos y vayan a la avanzada; por el contrario, el ser humano es un "sujeto" de estudio, y además de las implicaciones éticas que presentaría su manipulación y experimentación, existe una consideración ontológica fundamental: es libre, y la libertad no lo convierte en un sujeto caótico, incomprensible y dejado al azar, sino en una entidad misteriosa, capaz de certeza dentro de cualquier incertidumbre.

\section{INCOMPLETITUD E INCONSISTENCIA DE LOS SISTEMAS FORMALES (K. GÖDEL)}

Kurt Gödel ${ }^{1}$ nació Brno, Moravia en 1906, y vivió en Viena hasta 1940; en 1930 se doctoró en la Universidad de Viena, curiosamente con una tesis de apenas once páginas, que demostraba la completitud semántica del cálculo de primer orden, es decir, que cada verdad lógica es probable. Hacia 1931 escribió una de las obras revolucionarias dentro de la historia de las matemáticas: Sobre las Sentencias Formalmente Indecidibles de Principia Mathematica y Sistemas Afines («Über formal unentscheidbare Sätze der Principia Mathematica und verwandter Systeme In) $)^{2}$. En ella observó que, dentro de un sistema, existen proposiciones que versan sobre la consistencia del sistema mismo y que son de suyo indecidibles.

\footnotetext{
${ }^{1}$ Su biografía se construyó a partir de las siguientes fuentes: Gran Enciclopedia Rialp, T.XI, Rialp, Madrid 1972; Encyclopedia of Philosophy, V. 4, Routledge, London-New York 1998; Enciclopedia Encarta (Versión 9.0.0.0710), Microsoft, Redmond 2000; Diccionario Enciclopédico Salvat, T. X, Salvat, Barcelona 1991. The Cambridge Dictionary of Philosophy, Cambridge U. Press 1999.

${ }^{2}$ Existe una reproducción de este ensayo en inglés en Yggdrasil's Library: www.ddc.net/ygg/etext/godel/godel3.htm
} 
De 1933 a 1939 fue Privatdozent en la Universidad de Viena, y publicó en el mismo período artículos sobre el problema de elección, la relación entre lógica clásica y lógica de intuición. Al mismo tiempo, ingresó al Círculo de Viena, del cual salió por el desacuerdo con su postura filosófica. Kurt Gödel pasó el período escolar de 1933-4 en el Instituto de Estudios Avanzados en Princeton. En 1940 emigró a Estados Unidos permaneciendo en Princeton, donde se relacionó cercanamente con su amigo A. Einstein. Murió en 1978, a causa de desnutrición, tal vez por sus problemas mentales y ciertas fobias que le aquejaron al final de su vida.

Es necesario, al hablar de Gödel, mencionar la obra Principia Mathematica $(P M)$ de Alfred Whitehead y Bertrand Russell. En sus obras completas, Kurt hace copiosas referencias a la obra de Russell, ésta constituía un baluarte en la historia de la Lógica, ya que con pocos símbolos y realmente un mínimo de axiomas y teoremas, construía todo el edificio de la matemática, augurando así la posibilidad de una construcción formal (lógica) de todas las ciencias, al modo de $P M$.

Los signos utilizados en $P M$-utilizados posteriormente por el mismo Gödel para llegar a resultados sorprendentes- son:

\begin{tabular}{|c|c|c|}
\hline$\neg$ (Negación "no") & $\Lambda$ (Conjunción "y") & $\forall \mathrm{x}($ Para toda $\mathrm{x})$ \\
\hline v (Disyunción "O") & $\equiv($ Igual $\mathrm{a} \ll=»)$ & $\exists \mathrm{x}$ (Existe $\mathrm{x}$ tal que $)$ \\
\hline $\begin{array}{l}\rightarrow \text { (implica, } \\
\quad \text { se sigue que) }\end{array}$ & $\begin{array}{c}\in(\text { pertenece } \mathrm{a}, \\
\text { miembro })\end{array}$ & $\begin{array}{c}\subset \text { (contiene } \mathrm{a}, \\
\text { conjunto })\end{array}$ \\
\hline
\end{tabular}

Por ejemplo, el principio aristotélico de no-contradicción, en un lenguaje formal equivaldría a: $\neg(\mathbf{A} \Lambda \neg \mathbf{A})$; o el de identidad a: $\mathbf{A} \equiv \mathbf{A}$

La intención russelliana, muy pitagórica, suponía la simplificación de las verdades científicas a fórmulas consistentes o coherentes dentro de un sistema formal. A este afán se sumaba el formalismo de Zermelo y Fränkel, y los axiomas de los números naturales de Peano. 
Los teoremas de Gödel tendrán dos reproches a los conceptos anteriores:

a) No hay sistemas formales consistentes ${ }^{3}$.

b) No hay sistemas formales completos ${ }^{4}$.

Quizá la mejor manera de introducir los teoremas de Gödel sea con este sencillo ejemplo, que la literatura científica consagró como la "máquina de Gödel":

Imaginemos que existe una computadora capaz de contestar cualquier pregunta; después se escribe la frase $G$-de Gödel— «esta computadora nunca dirá que esta frase es verdadera". Ahora se le pregunta a la computadora: «Es G verdadera o falsa?" Si la computadora contestara que es verdadera, entonces el enunciado $G$ sería falso, y la computadora se equivocaría al decir que era verdadero, por tanto, no podrá decir nunca que el enunciado es verdadero. Entonces, el enunciado $G$, que afirma: «esta computadora nunca dirá que esta frase es cierta" es verdadero y la computadora no puede detectarlo así. Hay, entonces, por lo menos, una verdad que la computadora no puede pronunciar, aunque nosotros sí lo sepamos. En otras palabras, la computadora no es capaz de contestar cualquier pregunta que se le formule.

Si se traslada esto al terreno científico, y la máquina representa las matemáticas, podemos imaginar el duro embate que representó Gödel para quienes vislumbraban en esta ciencia el modelo para todas las demás o su fundamento5; en concreto, para Hilbert y su formalismo, que pretendía hacer de la matemática clásica, de sus conceptos e ideas, un sistema de signos gráficos manipulables, demostrables, combinables conforme a reglas mecánicas arbitrarias ${ }^{6}$.

\footnotetext{
${ }^{3}$ Se entiende por consistencia, la propiedad de un sistema que afirma que para todos sus enunciados hay una clasificación en "verdadero" o "falso".

${ }^{4}$ Se entiende por completo o completitud, la propiedad de un sistema que señala que puede probarse, dentro del sistema y con los axiomas del mismo, cualquier enunciación contenida en el sistema.

${ }^{5}$ Históricamente existieron pretensiones similares al formalismo lógico de Hilbert, basta recordar la teoría de los números de Pitágoras, la Ethica ordine geometrico demonstrata de Baruch Spinoza, o al afán por los juicios sintéticos a priori de las ciencias físico-matemáticas de Kant.
}

${ }^{6}$ Cfr. GÖDEL K., Obras Completas, Alianza Editorial, Madrid 1981, p. 92-92. 
El primer teorema de Gödel puede resumirse así: para $\varphi$ (enunciado) existe un número "n" que tiene propiedades. Supongamos que una de esas propiedades es "no ser deducible del sistema", entonces habrá "n" sin prueba $\varphi^{7}$.

Más extensamente, el procedimiento gödeliano consiste en asignar un número natural a cada símbolo del sistema y utilizarlos como exponentes de números primos que después se multiplican obteniendo así, para cada fórmula del sistema, un número y sólo uno: su número de Gödel (NG). Por ejemplo, según la fórmula " $\mathrm{x} v \neg \mathrm{X}$ " $\mathrm{x}=1, \mathrm{v}=2,\urcorner=3$, y la serie de números primos es: 2,3,5,7,11...; asignando el exponente la serie de números primos, la fórmula se puede leer así: “2 $2^{1} \cdot 3^{2} \cdot 5^{3} \cdot 7^{1 \text { " }}$ y equivale al número 15,750. Gödel formuló enunciados indecidibles $^{8}$, similares al del ejemplo de la computadora, los convirtió en una multiplicación de números primos con sus respectivas potencias, y encontró que: jexisten fórmulas verdaderas —aritméticamente- cuyo NG no tiene un similar en lógica formal, es decir, no tiene prueba, no es demostrable en el sistema! En otras palabras, enunció su primer teorema, también llamado teorema de la incompletitud: cualquier sistema formal es incompleto, pues existen en él proposiciones indecidibles.

Derivado de su primer teorema está el segundo, llamado teorema de la inconsistencia ${ }^{9}$, que es al que directamente alude el ejemplo de la "máquina de Gödel". En un sistema $S$, no puede comprobarse la consistencia (falta de contradicción)

\footnotetext{
${ }^{7}$ En una nota complementaria añadida en 1963 al ensayo Sobre las Sentencias Formalmente Indecidibles de Principia Mathematica y Sistemas Afines, Gödel escribió: "Se puede probar rigurosamente que en cada sistema formal consistente hay sentencias aritméticas indecidibles y que, además, la consistencia de cualquiera de esos sistemas no puede ser probada en el sistema mismo" (GÖDEL K., o.c., p.89).

${ }^{8}$ Porque el sistema no puede decidir por sí mismo la verdad o falsedad del enunciado.

${ }^{9}$ La enunciación original se encuentra en el Teorema XI de su ensayo: Sobre las Sentencias Formalmente Indecidibles de Principia Mathematica y Sistemas Afines: "Sea K una clase recursiva primitiva y consistente cualquiera de fórmulas. Entonces ocurre que la sentencia que dice que $\mathrm{K}$ es consistente no es K-deducible" (GÖDEL K., o.c., p.87).
} 
del mismo. Es decir, los axiomas del sistema no pueden probar la consistencia del sistema mismo, pues son parte de él; se tendría que recurrir a axiomas ajenos del sistema, que al incluirse al mismo, formaría un sistema mucho mayor que el inicial y que, a su vez, no podría probar su consistencia. Como indica el procedimiento tomista para la demostración de la existencia de Dios: "no se puede ir al infinito", en este caso, en la serie de axiomas ajenos al sistema para probar la consistencia del sistema mismo. Surge, por tanto, una consecuencia obligada y dolorosa: los sistemas formales de la ciencia no son consistentes, no pueden decir y decidir de sí mismos su falta de contradicción.

Respecto a la recurrencia en matemáticas a axiomas superiores para demostrar problemas del sistema, pensemos en por qué cualquier número dividido entre cero es igual a infinito, o cualquier número elevado a potencia cero es igual a uno. Son problemas que no pueden ser resueltos del mismo modo que se resuelven el resto de ellos, de ahí la necesidad de recurrir a explicaciones más complejas, que implican salirse de los axiomas y teoremas propios del caso.

Los teoremas gödelianos se han utilizado para combatir el formalismo y el logicismo; no obstante, también inspiran a filósofos que han visto en ellos la respuesta a fenómenos epistémicos, como lo hicieron Myhill y Reinhardt. También Gödel alentó a filósofos para combatir metafísicamente el materialismo y las doctrinas de filosofía de la mente que identificaban a ésta con el funcionamiento de una computadora; por una parte quedaba probado que la mente humana no era codificable logarítmicamente como una computadora (con lo que evitaba la aplicación de sus teoremas de incompletitud e inconsistencia a lo humano). Respecto a los argumentos antimecanicistas fundados en Gödel encontramos a Lucas, Benacerraf, Penrose, cuyos argumentos se sintetizan así: 1) si la mente humana fuera mecanizable, entonces, de acuerdo al Teorema de Gödel, no podría saber si sus 
creencias son consistentes; 2) la mente humana sabe que sus creencias son consistentes; 3) la mente humana no es mecanizable ${ }^{10}$.

Quiero adelantar, aunque posteriormente la reflexión final lo hará, la fractura epistemológica y metodológica que suponían los teoremas de Gödel. Por una parte, colocaban en tela de juicio los fundamentos de los sistemas científicos, al igual que sus hallazgos y técnicas, y por otra, abrían un campo de incertidumbre — con cierto sabor a melancolía - respecto al saber humano, a sus límites y posibilidades, que ya eran ciertos e indudables.

\section{EL PRINCIPIO DE INCERTIDUMBRE (W. HEISENBERG)}

Werner Karl Heisenberg ${ }^{11}$ nació en Wurzburg, en 1901. Estudió física en la Universidad de Munich. Obtuvo su doctorado en 1923. Durante el año siguiente estuvo como ayudante de Max Born en Gotinga. Se trasladó a Copenhague para trabajar con Neils Born y allí fue miembro fundador del Instituto Copenhague para la interpretación de los fenómenos cuánticos, Instituto que después realizará una interpretación sui generis del Principio de Incertidumbre del mismo Heisenberg. De 1927 a 1941 dictó la materia de Física teórica en la Universidad de Leipzig, en Alemania. En 1927 estableció el Principio de Incertidumbre, que enuncia que en la Mecánica cuántica es imposible conocer simultáneamente el valor de dos variables: posición e impulso. En 1930 publicó Principios de la Teoría Cuántica (Die Physikalischen Prinzipien der Quantentheorie) que le valió dos años más tarde, junto con sus logros de 1927, el premio Nobel de Física. En 1948 fue

\footnotetext{
${ }^{10}$ Cfr. Encyclopedia of Philosophy, voz: Gödel, V. 4, Routledge, London-New York 1998.

${ }^{11}$ Su biografía se construyó a partir de las siguientes fuentes: Gran Enciclopedia Rialp, T.XI, Rialp, Madrid 1972; Encyclopedia of Philosophy, V. 4, Routledge, London-New York 1998; Enciclopedia Encarta (Versión 9.0.0.0710), Microsoft, Redmond 2000; Diccionario Enciclopédico Salvat, T. XI, Salvat, Barcelona 1991. The Cambridge Dictionary of Philosophy, Cambridge U. Press 1999.
} 
nombrado director del Instituto Max Planck. Para algunos de sus biógrafos, Heisenberg estuvo involucrado en la producción bélica atómica de la segunda guerra mundial, sin embargo él mismo se desdijo de esas acusaciones. En 1969 escribió su autobiografía intelectual: Más allá de la Física (Der Teil und das Ganze). Murió en Munich en 1976.

La mecánica cuántica, movimiento en el que se inscribe Heisenberg, tuvo lugar entre 1923 y 1927, era una nueva forma de concebir los fenómenos físicos, resolviendo los problemas que la física clásica de Newton dejaba sin respuesta. Max Planck, físico alemán, desarrolló la teoría de los cuantos o paquetes de energía, que son emitidos o absorbidos por la materia, y que daban respuesta a problemas de termodinámica, radiación y electricidad.

Planck descubrió que la radiación de un cuerpo es equivalente a $h u$, donde $u$ es la frecuencia de la radiación multiplicada por ћ, que era una constante o cuanto de acción. La teoría newtoniana, por ejemplo, no podía explicar por qué, en un laboratorio, un metal emitía la misma cantidad de electrones incidiendo en ella diversos tipos de radiaciones; pero la teoría cuántica sí resolvía este "fenómeno fotoeléctrico", pues no era la intensidad sino la frecuencia de la radiación la que determinaba la emisión de electrones de un cuerpo.

Quedaron, no obstante, problemas experimentales por resolver dentro de la incipiente mecánica cuántica de Planck. Dos fueron las grandes formulaciones posteriores a Planck que intentaron sistematizar su teoría: la mecánica ondulatoria de Schrödinger, y la mecánica de matrices, encabezada por Heisenberg.

Heisenberg, junto con Pascual Jordan, elaboró, pues, una nueva teoría cuántica, distinta de la de Schrödinger, de tintes ondulatorios, por una matricial. Esta teoría servía para la comprensión de los fenómenos atómicos y sus relaciones (atracciones, repulsiones, efectos electromagnéticos y fotoeléctricos, etcétera); sin embargo, al estar experimentando, Heisenberg descubrió 
algo más importante que la descripción de los movimientos de las partículas atómicas, notó que el observador siempre supone una interferencia en el movimiento y posición de las partículas que estudia; es decir, sí se puede determinar la posición de una partícula en el espacio o el momento ${ }^{12}$ (energía de impulso), pero no ambas al mismo tiempo.

Por ejemplo, para determinar la posición de una partícula se emite luz para que choque con la partícula, y el impacto producido nos dirá el lugar específico donde ocurrió tal fenómeno. Sin embargo, como Planck lo había probado, la luz también se comporta de manera ondulatoria y corpuscular, es decir, también es un cuerpo, y tiene a su vez, una posición y un momento determinado, de ahí que el choque del fotón (haz de luz) con la partícula, transmitirá parte del momento del fotón a la partícula, alterándola en su velocidad.

Por otra parte, como la longitud de onda de la luz es inversamente proporcional a su momento, y se utiliza en el experimento antedicho luz con una longitud de onda pequeña, su momento será mayor, así que la colisión lo será también, y la precisión en la medición de la posición será casi total, sin embargo, el impulso transmitido a la partícula será muy elevado y crecerá, por ende, la indeterminación de su momento. Si por el contrario, se utiliza una luz con una longitud de onda muy grande - y momento mínimo-, no habrá casi afectación al momento de la partícula, lo cual podemos calcular con certeza, sin embargo, no habrá colisión, quedando indeterminada la posición de la partícula.

Resulta, entonces, un margen obligado de incertidumbre en el conocimiento del microcosmos, que jamás llegará a ser cero, quedando la física en un suelo distinto de la matemática exacta: el de la probabilidad.

Asimov proponía un ejemplo muy simple al Principio de Incertidumbre y la injerencia del observador en la alteración

${ }^{12}$ Momento o impulso es el resultado de la masa por la velocidad de una partícula. 
del fenómeno a ser medido: si alguien quiere saber la temperatura de un líquido, meterá un termómetro, pero la temperatura que posee el termómetro es distinta a la del líquido, así que distorsionará, aunque sea mínimamente, la temperatura a medir; así, nunca se sabrá con exactitud la temperatura del líquido.

Pauli explicaba en términos científicos el principio de Heisenberg así:

"No es posible adscribir simultáneamente a un cuerpo material una posición exacta y un valor exacto de su momento, sea este cuerpo macroscópico o atómico, esté cargado eléctricamente como un electrón o sea eléctricamente neutro como un fotón, ${ }^{13}$

La fórmula matemática que expresa el Principio de Incertidumbre es:

$$
\Delta \mathrm{x} \Delta \mathrm{p} \geq \hbar / 2
$$

Donde $\Delta \mathrm{x}$ es la incertidumbre de la posición, multiplicada por $\Delta \mathrm{p}$ que es la incertidumbre del momento, que es mayor o igual a la constante de Planck ( $\hbar$ ) dividida entre dos.

Resta por considerar un aspecto de este principio. ¿La incertidumbre es del sujeto o del objeto? En otras palabras, ¿la incertidumbre es epistemológica u ontológica?

En un inicio, Heisenberg optó por la primera posibilidad, pues la imprecisión residía en la medición y no en la marcha natural de las partículas que, dejadas al flujo natural, llegarían seguramente a una posición $x$ con un momento $y$, aunque desconocidas por el espectador. De hecho, Albert Einstein tomará partido por esta postura, que encaja con su teoría de la relatividad — del espectador — respecto al fenómeno, y no del fenómeno mismo; lo mismo sucederá con la interpretación de Copenhague.

13 PAULI Wolfgang, Escritos sobre Física y Filosofía, Debate, Madrid 1996, p. 44. 
"Al explorar este problema, Heisenberg ofreció una imaginativa explicación de la incertidumbre, considerándola como indicadora de una limitación fundamental de nuestra capacidad para fijar todas las propiedades de un sistema a un grado arbitrario de precisión por cualquier técnica experimental. La idea básica aquí era que cualquier medición realizada sobre un sistema debe, inevitablemente, perturbar físicamente al sistema medido. El fijar una cantidad a un grado determinado de precisión perturbaría, pues, el sistema de forma tal que nuestro conocimiento del valor de alguna cantidad conjugada quedaría reducido, pudiendo ésta tomar, después de la medición, cualquier valor de un gran intervalo de valores" ${ }^{14}$.

No obstante, tras diálogos con Born, Heisenberg trasladó sus hallazgos al comportamiento de las partículas y no tanto a la incertidumbre que producía la injerencia del observador. La nueva idea era comprender que las partículas en sí mismas son inciertas ${ }^{15}$, puesto que la complejidad del mundo impone relaciones obligadas a las partículas, creándose así una red de interrelaciones que afectan en cada instante la posición y momento; por ejemplo, el amanecer, el canto de un pájaro, un trueno o el estornudo de un niño, afectarían y distorsionarían constantemente el movimiento de las partículas.

Por eso comenta Sklar: "Heisenberg se vio inducido a aceptar la lectura bohriana ontológica, más radical, de la incertidumbre. Esto es, aceptó la idea de que la incertidumbre refleja la dispersión irreducible de características del sistema, no meramente una limitación de nuestro conocimiento de propiedades conjuntas a grados arbitrarios de precisión ${ }^{16}$.

14 SKLAR Lawrence, Filosofía de la Física, Alianza Universidad, Madrid 1994, p. 257.

${ }^{15}$ Para profundizar más sobre la cercanía entre la incertidumbre de Heisenberg y el noúmeno kantiano, se puede consultar su ensayo: "La mecánica cuántica y la filosofía de Kant" contenido en su libro: Diálogos sobre Física Atómica, Universidad Autónoma de Puebla, Puebla 1998.

${ }^{16}$ Sklar Lawrence, o. c., p.259. 
Al igual que los teoremas de Gödel, el Principio de Incertidumbre de Heisenberg, representó un duro golpe al racionalismo y al positivismo, en cualquiera de sus formas. Claro está, acompañados de ellos estaban Planck y su teoría cuántica, Einstein y la relatividad del universo, las teorías de entropía que postulaban la paulatina pérdida de calor en los cuerpos, que hacían ver a la humanidad, no sólo la contingencia del conocimiento humano, sino la contingencia de los fenómenos naturales — de alguna manera libres-, no sujetos a las leyes interpretativas humanas; hacían replantear la misión de la ciencia como una humilde intérprete de un mundo complejo, dinámico e inconmensurable.

Las consecuencias de las doctrinas cuánticas, en especial la matricial de Heisenberg, no se hicieron esperar: no existe un determinismo físico, mucho menos científico. La física se convierte en una teoría, más o menos probable, de los fenómenos que ocurren en el universo. El mito de Laplace ${ }^{17} \mathrm{y}$ otros científicos caían por tierra: si el presente es complejo, el futuro se vuelve, literalmente, incierto; la forma de concebir el mundo era ya en otra dirección ${ }^{18}$.

\section{EL MISTERIO EDUCATIVO (SAN AGUSTÍN)}

Considero que las consecuencias de la teoría de la incompletitud de los sistemas formales, al igual que el principio de

${ }^{17}$ En su Ensayo Filosófico sobre las Probabilidades afirmaba: "Si concebimos una inteligencia que en un instante determinado abarcara todas las relaciones entre todos los entes del universo -una inteligencia lo suficientemente amplia que permitiera someter estos datos al análisis-, ésta podría establecer las posiciones respectivas, el movimiento y las propiedades generales de todos estos entes, desde los mayores cuerpos del universo al menor de los átomos; para ella nada sería incierto y el futuro así como el pasado estarían presentes ante sus ojos", apud: GIRÓN González F. J., "Determinismo, caos, azar e incertidumbre", http://www.fsanmillan.org

${ }^{18}$ Cfr. HEISENBERG Werner, Physics \& Philosophy. The Revolution in Modern Science, Harper, New York 1963, p.187: "The philosophical implications of modern physics have been discussed in the foregoing chapters in order to show that this mos modern part of science touches very old trends of thought at many points, that it approaches some of the very old problems from a new direction". 
incertidumbre, realmente hirieron a profundidad el pensamiento científico humano. No es que hayan constituido algún mal para la ciencia, al contrario, son avances en el conocimiento, aunque esos avances aparentemente consistan en la imposibilidad de avanzar; más bien, se han extrapolado las inferencias de los teoremas de Gödel y Heisenberg a las ciencias de lo humano, identificando el comportamiento atómico o sistémico-matemático con el psíquico o social humano. Por ejemplo, la Teoría del Caos $^{19}$, heredera de la "incertidumbre", ha sido indebidamente transpolada a fenómenos sociales como movimientos migratorios, natalidad/mortandad u oficios concretos en que se emplean las personas. Parece que la complejidad del mundo humano es idéntica a la complejidad (incierta-probable) del mundo físico.

Una clara aplicación de la Teoría del Caos en las situaciones humanas está descrita por Balandier así: "Si los científicos parecen jugar al caos sobre el gran tablero de la naturaleza, los analistas de la modernidad se lanzan con menos probabilidades a un juego similar. A éste lo he definido no hace mucho con una fórmula: el movimiento más la incertidumbre. El primero de esos dos términos se realiza en formas múltiples, vistas por muchos como otras tantas ocultaciones o máscaras del desorden. El vocabulario posmodernista se ajusta a este inventario exploratorio de la "deconstrucción" y de las simulaciones. Hace unos años se estableció progresivamente la cuenta de las desapariciones: desde el campo hasta la ciudad, desde los grupos hasta las relaciones entre individuos, desde estos últimos hasta los espacios de la cultura y el poder, todo ha sido condenado a desaparecer... El segundo término de mi fórmula - la incertidumbre- expresa a la vez irrupción de lo inédito bajo los impulsos de la modernidad, y el riesgo, para el hombre, de encontrarse en posición de exiliado, extranjero

19 Podemos encontrar algunos ejemplos en la teoría de la différance y la deconstrucción de Derrida, los rizomas y las caoideas de Deleuze y Guattari, y el Desorden de Balandier. 
o bárbaro, en su propia sociedad, si la incomprensión de lo que está en vías de hacerse lo alejase de una civilización contemporánea donde sólo identificara el caos y el no sentido ${ }^{20}$.

Hay dos fuertes cuestionamientos qué hacer a estas posturas: ¿es justificada, metodológicamente, la analogía entre los sucesos físico-matemáticos y los humanos?, y ¿necesariamente las conclusiones de la incertidumbre epistemológica-ontológica de las partículas atómicas (micro) determinan un comportamiento caótico y extraño en el ser humano (macro)?

De entrada rechazo cualquier univocidad entre las conclusiones físico-matemáticas y las de las ciencias de lo humano, ya en lo micro, como lo son las partículas subatómicas, ya en lo macro como los comportamientos corpusculares de las galaxias, por el simple hecho de la irreductibilidad del ser humano a mera materia. Sin embargo, este argumento, que escapa del objeto de estudio actual, no necesariamente concluiría la no existencia del caos en las situaciones humanas, pues aun aceptando la presencia del espíritu, eso no establece, per se, el orden y la certeza de los fenómenos antropológicos.

Más bien, hay que emprender la vía de solución a este problema analizando, por una parte, el acto educativo según el agustinismo, para descubrir en él la similitud con la incertidumbre que propugnaba Heisenberg, y por otra parte, esbozar la noción de "misterio" educativo, para encontrar la desemejanza con las nociones de incertidumbre, incompletitud e inconsistencia, que actualmente se abarcan en la de "caos".

El De Magistro ${ }^{21}$ agustiniano comienza con una pregunta previa a toda cuestión pedagógica: ¿para qué sirve el lenguaje? A lo cual responde: "O para que enseñemos o para que recordemos", que se reducen a un solo acto: enseñar, pues

${ }^{20}$ BAlandier G., El Desorden. La Teoría del Caos y las Ciencias Sociales. Apud: GUTIÉRREZ Pantoja G., Metodologia de las Ciencias Sociales II, Oxford U. P., México 1998, p.296-7.

${ }^{21}$ SANABRIA J. R. y BEUCHOT M., Del Maestro. San Agustín de Hipona y Del Maestro Sto. Tomás de Aquino, UIA, México 1990. 
incluso aprendiendo o recordando exponemos o manifestamos —enseñamos - algo a nuestro interlocutor. Esta primera respuesta encubre ya el problema filosófico de la pedagogía: ¿es simplemente que, hablando, el maestro transmite los conocimientos al alumno? Pero si el lenguaje expresa ideas, y las ideas expresan cosas, ¿cómo es posible que el lenguaje exprese y transmita la realidad? Esta antinomia entre palabra y realidad, Agustín la resuelve diciendo que las palabras son signos de las cosas, y como tales presuponen el contacto directo, el conocimiento previo de las cosas para después ser entendidas; así se expresa en el capítulo X: "Aprendo que la palabra es un signo cuando encontré de qué era el signo: lo que aprendí no fue por el significado, sino por la visión de la cosa. Por lo que, más bien, conocida la cosa se aprende el signo, que, dado el signo, se aprende la cosa ${ }^{22}$.

Es en la anterior tesis que se manifiesta la preocupación de San Agustín: aprendemos cosas por medio de signos o comprendemos los signos gracias a las cosas. El obispo se inclinará en su síntesis, a las dos fórmulas, y así dice en el capítulo XI: "Quien enseña algo es el que presenta a mis ojos, o a cualquier sentido del cuerpo, o también a la misma mente, lo que quiero conocer"; indica, asimismo, que nada se puede conocer sin signos, pues mostrar algo simplemente, no es mostrar su esencia, sino muchas veces su accidentalidad, lo cual no produce un conocimiento completo de la cosa; pero, a su vez, ningún signo — sensible y material— es capaz de

\footnotetext{
${ }^{22}$ Recordemos que "enseñar" proviene de dos palabras latinas "in signum": proponer un signo a alguien para que aprenda. $\mathrm{Y}$ es que el fenómeno educativo precisa de signos o instrumentos para llevar a cabo su fin: la optimización del hombre, ya en su aspecto intelectual ya en el moral. Al respecto, Casotti afirma que la columna vertebral del problema educativo a lo largo de la historia consiste en la admisión de una quaestio facti: existe la educación, y en la indagación de una quaestio iuris: ¿cómo es posible la educación? Pues bien, la condición de posibilidad de la educación se revela a través del acto mismo de enseñar, que necesita de signos para llevarse a cabo. Cfr. CASSOTI M., "De Magistro" di S. Agostino e di S. Tommaso", en: AGAZZI A. et al., Questioni di Storia della Pedagogia, La Scuola Ed., Brescia 1963, p.122-123.
} 
indicar por sí mismo y suficientemente lo más esencial de las cosas, que es inmaterial, y que, constituyendo lo valioso de la enseñanza, no se recibe por palabras, gestos o demostraciones. O, para decirlo con más claridad, la acción humana se vería reducida a pura enseñanza ( in signum $=$ proponer signos) sin llegar a ser verdadera educación.

Educar entraña, sin embargo, algo mucho más profundo que la enseñanza, implica crecimiento, madurez, adquisición de virtudes, autonomía, aprecio de la cultura, colaboración al bienestar de la comunidad, comunicación efectiva y afectiva, en fin, una plenificación del ser humano. Esto es lo que vislumbró San Agustín, y que a partir del fenómeno de la enseñanza no se puede dar explicación al fenómeno educativo. Educar es más, mucho más, educar es ante todo un misterio.

Para aproximarnos a la entidad del misterio, San Agustín propone dos características fundamentales, cuando describía el Mysterium tremendum ac fascinans ("Misterio tremendo y fascinante $)^{23}$. Tremendo, porque causa miedo, porque es tan grande y supera en mucho la capacidad de conocimiento, que se vuelve digno de respeto o hasta reverencia. Y no me refiero a grande en cuanto su magnitud física o la complejidad de su estructura, sino grande en "dignidad", excesivo en "belleza" ${ }^{24}$. Fascinante, porque seduce irresistiblemente, porque atrae como un imán, porque no se puede no considerar.

¿Donde se encuentra el misterio? ¿Qué es a la vez tremendo y fascinante? La persona. Esta respuesta no es ofrecida sin más, sino que puede ser corroborada por cualquiera; en efecto, ¿a quién le atrae irrecusablemente el momento de una partícula atómica?, y sin embargo, a todos nos fascina el ser humano, nuestra madre o padre, nuestro mejor amigo, la esposa o un

\footnotetext{
${ }^{23}$ Ciertamente San Agustín intentaba dilucidar la entidad del misterio divino, aunque con toda razón se pueden aplicar estas propiedades - no en grado sumo- a los demás seres espirituales.

${ }^{24}$ San Agustín fue claro al diferenciar la magnitud (corpórea) y la grandeza, referida a realidades superiores, como el alma o la virtud. De Trin. IX, 4,4: "Et vita quaelibet quolibet corpore maior est, non mole, sed vi».
} 
hijo, en fin, un educando; a quién no le maravilla el proceso educativo que experimenta un niño desde su nacimiento hasta que aprende a hablar, expresarse, ser agradecido, etcétera.

Como se expuso, el misterio es tremendo, lo cual le otorga una excelencia respecto a la facultad cognitiva que lo trata de interpretar, aquí ciertamente está el gozne con la doctrina de Heisenberg, pues existe una real incertidumbre de las capacidades humanas ya que no pueden ni podrán agotar la inmensidad del misterio. Sin embargo, el misterio también es fascinante, de ahí que la libertad se empeñe en buscarlo, tenerlo y amarlo, y así, comprehenderlo (cum-prehendere, tomar alrededor, acoger, abrazar) aunque sin agotarlo (en juicios analíticos).

Así pues, si cada objeto de estudio de una ciencia determina su método de estudio, el misterio educativo humano debe ser abordado desde una metodología única, que considere la libertad no como variable estadística de leyes, sino como la más esencial de las leyes humanas, a cuya luz se resuelven todas las estadísticas. El método agustiniano para comprehender el misterio se puede reconstruir así: creer-entender-amar.

\section{CREER: ACEPTACIÓN DEL MISTERIO}

Creer no necesariamente es un acto religioso, es un acto de la vida común, necesario, sobre el cual están fundados muchos conocimientos ${ }^{25}$. Pensemos en cuántas cosas no creemos acerca de nuestro pasado y nuestros antepasados, en cuántos asuntos nos fiamos de los demás o en cuántas cosas aprendidas en la escuela o en los libros no las hemos corroborado nosotros, sino que las creemos ciertas y verdaderas. Creer es, en suma, dar asentimiento a verdades, Credere est cum assensione cogitare ${ }^{26}$.

${ }^{25}$ Cfr. GILSON E., Introduction à l'étude de Saint Agustin, Vrin, París 1949, p.32-33: "La croyance n'est en effet qu'une pensée qui s'acompagne d'assentiment (...) La croyance est donc une démarche de la pensée tellement normale, qu'elle est la condition même de la famille et par elle de la société (...) De toute façon, croire est encore une certaine manière de savoir; c'est une connaissance de la pensée, comme l'est la science proprement dite dont elle ne diffère d'ailleurs que par son origine".

${ }^{26}$ SAN AGUSTÍN, De praedestinationem sanctorum, II, 5. 
Esta forma natural de creer tiene como fundamento el testimonio de otra persona que, con su autoridad, respalda la veracidad de lo creído. Si una madre dice a su hijo: "no vayas con ese hombre", el niño obedece, cree, aunque no sepa por qué su madre le advierte aquello. El mismo San Agustín advirtió que, si sospechásemos de todos los conocimientos aceptados por fe, no quedaría intacto vínculo alguno de la sociedad humana $^{27}$. ¿Qué deberes — por ejemplo— habría para con unas personas de las que no me consta que son mis padres?

Sin embargo, ése es un creer que... es decir, es un creer algo. Existe, además, un creer más profundo y comprometedor: creer en, creerle $a$. Es éste el creer o fe que versa sobre el misterio, sobre la totalidad de la persona y no sobre la parcialidad de sus conocimientos, advertencias o consejos. Si se cree $a$, se cree entonces todo, se le cree a la persona, se fía del misterio.

"Creer es sabiduría, el acto por el que reconocemos no poder conocerlo todo directamente. La creencia no es obstáculo para el conocimiento, antes bien, lo aumenta cuando es claro y está bien fundado, o sea, es creíble» ${ }^{28}$.

El acto epistemológico de la fe supera el margen de dudas e incertidumbres que naturalmente surgirían al conocer el misterio. Se cree en alguien, se le cree totalmente; se asume una posición de acogida y aceptación de la totalidad, aunque haya un desconocimiento de las partes que la componen. Aquí comienza ya la fractura epistemológica con la incertidumbre de Heisenberg, pues mientras la ciencia tiene un conocimiento totalmente cierto de la parcialidad e incomprensible —hasta caótico — de la totalidad, la fe en alguien, o sea, creer en el misterio de la persona, alcanza un conocimiento incierto en la parcialidad, pero coherente y consistente en la totalidad. En todas sus obras San Agustín se muestra claro

${ }^{27}$ SAN AGUSTÍN, De utilitate credendi, c.12, n.26: "Multa possunt afferri, quibus ostendatur nihil omnino humanae societatis incolume remanere, si nihil credere statuerimus, quod non possumus tenere perceptum".

${ }^{28}$ SCIACCA M. F., San Agustín, Luis Miracle, Barcelona 1955, p.305. 
sobre la impenetrabilidad (cognitiva) de los misterios en esta vida, pero a la vez, se muestra igual de claro en lo asequible del misterio a la voluntad humana.

Un ejemplo educativo clarificará la fe como aceptación del misterio. Las normas éticas son enseñadas a un niño y él las asume por un acto de fe. Cree en sus padres, cree en que quieren su bien y no su mal, por eso le piden un comportamiento idóneo. En el niño no existe un exhaustivo análisis de las normas, es más, ni siquiera uno mínimo, y no es por la congruencia interna de esas normas por lo que las asiente, sino porque cree en sus padres. Así comienza un verdadero crecimiento ético, una madurez en la civilidad, que, sin duda, es educación.

\section{ENTENDER: PROFUNDIZACIÓN DEL MISTERIO}

Para comprender la relación entre el entendimiento y la fe, San Agustín pone un ejemplo muy sencillo: por qué alguien cree una frase que suena interesante y lógica y no cree otra absurda o tonta, si ambas provinieran de la misma persona; si el acto de creer no impulsara al entendimiento a comprender lo creído, no habría explicación al ejemplo. Y es que la sola autoridad no basta para sostener la creencia, es necesario cierto entendimiento de lo creído, al menos, que aquello no sea contradictorio o irracional.

El adagio agustiniano reza así: Intellige ut credas, crede ut

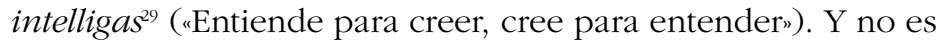
una doble fase de un acto, sino la constante interacción de dos formas de ir al misterio: creer y entender, entender y creer, y así sucesivamente, siempre con una profundización mayor. El misterio, que de suyo es inagotable, precisa de este movimiento dialéctico ascendente en aquel que quiere conocerlo.

${ }^{29}$ Epistola CXX, I, 3. En un comentario que Sciacca hace de esta frase, ubicándola en el contexto religioso, explica que se piensa para creer "la palabra humana", y así creer para entender la "Palabra de Dios": intellige ut credas verbum meum, crede ut intelligas verbum Dei. SCIACCA M. F., o.c., p.311, cita 25. 
El alumno, por ejemplo, cree en las palabras de su maestra, después las razona y ve que son lógicas y coherentes con lo que cotidianamente escucha y ve; con esta corroboración se acrecienta la confianza de él hacia ella, y con más asentimiento le cree, para nuevamente razonar lo creído hasta alcanzar una fe madura que sabe razonar lo que cree, y un entendimiento humilde, que necesita creer porque no alcanza a razonarlo todo por sí solo.

Tal vez en castellano la palabra "entender" nos dice mucho menos de la función de la inteligencia que el vocablo latino "intelligere, pues intus legere, leer dentro, implica la profundización, la penetración "cada vez" mayor del misterio

\section{AMAR: CERTEZA EN EL MISTERIO}

La tercera y fundamental de las fases de comprehensión del misterio, en la que se resuelve cualquier incertidumbre y se disipa cualquier duda, es el amor. Se entiende amor como la relación libre en que dos personas se comprometen al bien mutuo; es entonces razonable considerar la educación como una forma de amor, de ahí que cualquier deducción del fenómeno amatorio sea atribuida, con todo derecho, al educativo.

Fe y entendimiento, no bastarían por sí solos para dar cuenta de la comprehensión del misterio. Es necesario que ambos se corroboren existencialmente, que se vivan y verifiquen. Ante el misterio, es decir, ante la persona, la verificación no es de carácter experimental o manipulable, es una verificación libre, creída y razonada. ¿Cómo, sin embargo, surge la certeza dentro de un orden amatorio?

Cuando dos libertades se empeñan en amarse mutuamente, en procurarse el bien, entonces se crea un vínculo tal que proporciona un grado de certeza tanto a lo creído como a lo pensado. Regresemos a los ejemplos ofrecidos en el caso del creer y del entender. La certeza de la validez de las normas éticas propuestas a un hijo por su padre, son indudables y coherentes cuando el vínculo amatorio entre ellos es fuerte, 
pues el amor como condición, como situación en la que se cree y se reflexiona lo creído, da solidez a estos procesos y lo que no es alcanzado en certeza solamente por estos procesos, el amor lo proporciona. Así, el hijo se fía, con total seguridad, de que la advertencia "no vayas con ese hombre" es absolutamente cierta. Véase, por el contrario, la incertidumbre que produce esa misma frase si la dijera una persona desconocida. Lo mismo sucede con la relación maestra-alumno, esposoesposa, hermano-hermana, novio-novia, etcétera. El amor, como atmósfera existencial, proporciona certeza a los procesos epistemológicos, hace profundizar cada vez más y con mayor ímpetu en ellos ${ }^{30}$, y los llega a abarcar en su totalidad.

Sólo bajo esta óptica triple: creer-entender-amar, que no es estática, sino dinámica y en una constante retroalimentación se comprende la conclusión del De Magistro: "A quien ya, con su ayuda, tanto más ardientemente amaré cuanto más aprovecha en el estudio ${ }^{31}$, al igual que un hermoso pasaje del De libero arbitrio: "Si, pues, amamos y abrazamos asimismo con todo el afecto de nuestro corazón a esta nuestra buena voluntad — virtud por la que deseamos vivir justa y honestamente-, y la preferimos a todas las cosas que no podemos retener con nosotros, aunque queramos, síguese que moran en nuestra alma aquellas virtudes en cuya posesión consiste precisamente el vivir justa y honestamente, si realmente [es así] como la razón nos lo ha demostrado. De donde resulta que el que quiere vivir recta y honestamente, si realmente prefiere este querer a los bienes fugaces de la vida, conseguirá indudablemente ese tan inmenso bien, y con tanta facilidad cuanta supone el ser para él una misma cosa el querer algo y el tener lo que quiere,$^{32}$.

\footnotetext{
${ }^{30}$ Cfr. CASSOTI M., o.c., p. 131: "L'amore è quello che fa aprofondire l'uomo". ${ }^{31}$ SAN AGUSTÍN, Obras Completas, T. III, BAC, p. 755-756: "Tanto ardientius diligam, quanto ero in discendo provectior".

${ }^{32}$ SAN AGUSTÍN, Obras Completas, T. III, BAC, p.295.
} 
Concluyo, pues, con la profunda convicción que frente al misterio que suponen las personas, existe un espacio para la certeza y comprebensión, asequible sólo en la dinámica creer-entender, en ambientes de amor, respeto y valores. Si alguno aún me preguntara: “Existe entonces la incompletitud e incertidumbre en educación?», le contestaría con otra pregunta: “Esa "educación" de la que hablas, se da en el amor?». 


\section{BIBLIOGRAFÍA}

AGUSTÍN, San, Obras Completas, BAC, Madrid 1983.

CASSOTI M., "De Magistro" di S. Agostino e di S. Tommaso", en: AGAZZI A. et al., Questioni di Storia della Pedagogia, La Scuola Ed., Brescia 1963.

Diccionario Enciclopédico Salvat, Salvat, Barcelona 1991.

Enciclopedia Encarta (Versión 9.0.0.0710), Microsoft, Redmond 2000.

Encyclopedia of Philosophy, Routledge, London-New York 1998.

GILSON E., Introduction à l'étude de Saint Agustin, Vrin, París 1949.

GIRÓN González F. J., "Determinismo, caos, azar e incertidumbre, http://www.fsanmillan.org

GÖDEL K., Obras Completas, Alianza Editorial, Madrid 1981.

Gran Enciclopedia Rialp, Rialp, Madrid 1972.

GUTIÉRREZ Pantoja G., Metodología de las Ciencias Sociales II, Oxford U. P., México 1998.

HEISENBERG W., Diálogos sobre Física Atómica, Universidad Autónoma de Puebla, Puebla 1998.

HEISENBERG Werner, Physics \& Philosophy. The Revolution in Modern Science, Harper, New York 1963.

HOFSTADTER D. R., Gödel, Escher, Bach: Un Eterno y Grácil Bucle, Tusquets, Barcelona 1987.

HUlTGREN, G., Le Commandament d'amour chez Augustin: interpretation philosophique et theologique d'apres les ecrits de la période 386-400, Vrin, Paris 1939.

NAGEL E. y NEWMAN J. R., El Teorema de Gödel, Tecnos, Madrid 1970.

NAGEL, E., La Estructura de la Ciencia: Problemas de la Lógica de la Investigación Científica, Paidós, Barcelona 1991. 
PAULI Wolfgang, Escritos sobre Física y Filosofía, Debate, Madrid 1996.

POPPER K., El Universo Abierto. Un Argumento en Favor del Indeterminismo. Tecnos, Madrid 1986.

The Cambridge Dictionary of Philosophy, Cambridge U. Press 1999.

SANABRIA J. R. y BEUCHOT M., Del Maestro. San Agustín de Hipona y Del Maestro Sto. Tomás de Aquino, UIA, México 1990.

SCIACCA M. F., San Agustín, Luis Miracle, Barcelona 1955.

SKLAR Lawrence, Filosofía de la Física, Alianza Universidad, Madrid 1994.

TIRADO Benedi D., Antología Pedagógica de San Agustín, Fernández Editores, México 1966.

Yggdrasil's Library: www.ddc.net/ygg/etext/godel/godel3.htm 